\title{
ELASTIC SCATTERING FROM THE MULTIPERIPHERAL MODEL 饮
}

\author{
F.S. HENYEY \\ Physics Department, University of Michigan, Ann Arbor, Michigan 48104, USA
}

Received 6 June 1973

\begin{abstract}
Elastic scattering in the Multiperipheral Model (MPM) is examined by use of a new method for simplifying unitary calculations - multiparticle impact parameter. The $t$ dependence of elastic scattering in the MPM is related to the average transverse momentum of produced particles, the average multiplicities (observables), phase variation with transverse momentum, and clustering (not observables, in general). It is shown that MPM's are inconsistent with the combination of elastic and multiparticle data, unless either the cluster size or the phases have rather strong energy dependence.
\end{abstract}

Elastic scattering following from the Multiperipheral Model (MPM) by unitarity has been widely studied $[1-3]$. In this paper a new method, multiparticle impact parameter [4], is applied to this problem.

The multiperipheral model shares a number of features with the multiparticle data, such as limited trans. verse momentum, a spread of longitudinal momenta, etc. We will take these features from the data, so as to use as few details of the model as possible, and also so as not to allow the model to fit elastic scattering by means of a failure to fit multiparticle scattering.

There is, however, one feature of the MPM which is somewhat independent of the multiparticle data, and which distinguishes the MPM from other models which fit multiparticle data equally well. This feature is short range order. We will examine the effect short range order has on the elastic scattering.

Before turning to a simple MPM, we summarize the multiparticle impact parameter method [4]. The impact parameter, or transverse position, of the $j$ 'th produced particle is the variable cannonically conjugate, in a sense, to that particle's transverse momentum. (Notation: $\boldsymbol{b}_{j}=$ impact parameter, $\boldsymbol{k}_{j}=$ transverse momentum, $P_{j}=$ longitudinal momentum, $n=$ multiplicity.) The amplitude in impact parameter is given by

$$
\begin{aligned}
& \tilde{M}\left(b_{j}, p_{j}\right)= \\
& \quad \int \prod_{j=1}^{n} \frac{\mathrm{d}^{2} k_{j} \exp \left(\mathrm{i} k_{j} \cdot b_{j}\right)}{(2 \pi)^{2}} M\left(k_{j}, p_{j}\right) \delta^{2}\left(\sum_{j=1}^{n} k_{j}\right)
\end{aligned}
$$

Work supported by the U.S. Atomic Energy Commission.
The $p_{j}$ 's (longitudinal momenta) are not transformed. The inverse of this transformation is

$$
\begin{aligned}
& M\left(\boldsymbol{k}_{j}, p_{j}\right)=(2 \pi n)^{2} \\
& \quad \times \int \prod_{j=1}^{n}\left[\mathrm{~d}^{2} \boldsymbol{b}_{j} \exp \left(-\mathrm{i} k_{j} \cdot b_{j}\right)\right] \tilde{M}\left(\boldsymbol{b}_{j}, p_{j}\right) \delta^{2}\left(\sum_{j=1}^{n} b_{j}\right)
\end{aligned}
$$

Note the delta functions in (1) and (2). In terms of $\widetilde{M}$, unitarity for elastic scattering can be simply written in the high energy impact parameter approximation:

$$
\begin{aligned}
& \operatorname{Im} \vec{M}_{\mathrm{el}}(b)=\sum_{n} \frac{1}{2}(2 \pi)^{4}(2 \pi n)^{2} \\
& \quad \times \int \prod_{j=1}^{n} \mathrm{~d}^{2} b_{j} \frac{\mathrm{d} y_{j}}{4 \pi} \delta\left(\sum p_{j}\right) \delta\left(\sum_{\left.E_{j}-\sqrt{s}\right)}\right. \\
& \quad \times \delta^{2}\left(\sum_{b_{j}}\right) \delta^{2}\left(b-\sum_{j=1}^{n} b_{j} p_{j} / p\right)\left|\widetilde{M}\left(b_{j}, p_{j}\right)\right|^{2}
\end{aligned}
$$

$y_{j}$ is the rapidity, $\mathrm{d} y_{j}=\mathrm{d} p_{j} / E_{j}$. The first two delta functions express longitudinal momentum and energy conservation, the next two dimensional delta functions come from eq. (2), and the last one expresses angular momentum conservation. We only need to know the absolute value of $\widetilde{M}$.

We will first study a MPM with the shortest range order possible. This model is shown in fig. 1(a). The amplitude is assumed to factorize into a product of functions of the momentum transfers $t_{j}$. Each $t_{j}$ is given by a function of the $p_{j}$ 's and $E_{j}$ 's minus $Q_{j}^{2}$, 

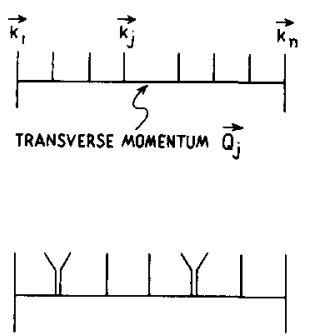

Fig. 1. a) The multiperipheral model with the shortest range order possible. $Q_{j}$ is a transverse momentum transfer. b) A modified multiperipheral model with correlations (clustering). Shown is a process with six steps, but with multiplicity minus one equal to eight.

where $Q_{j}$ is the transverse momentum transfer of the $j$ th link in the multipheral chain. There is supposed to be no further direct dependence of the amplitude on transverse momenta. For simplicity of calculation we take the dependence to be exponential in $t_{j}$, corresponding to a Gaussian in $Q_{j}$ although as we go along, it is easily seen that the results are insensitive to the functional form of the amplitudes' dependence on $Q_{j}$, but are only sensitive to one parameter of this dependence.

In fact, there is further, indirect, dependence of the amplitude on transverse momenta [5]. The amplitude depends on both energies and longitudinal momenta. These, of course, are not independent, but are related by $E_{j}^{2}-P_{j}^{2}=m^{2}+k_{j}^{2}$. Thus, for example, elimination of the energies in favor of the longitudinal momenta will introduce extra dependence on the transverse momenta. We shall ignore this extra dependence for two reasons. First, this indirect dependence on transverse momentum is always accompanied by dependence on longitudinal momentum. Therefore, if a transverse momentum becomes unfavorably large, the decrease in the matrix element can be somewhat compensated for by slightly different ratios of longitudinal momenta. Secondly, to the extent that the matrix element factorizes into a product of dependence on longitudinal and transverse momenta, the amplitude in impact parameter space will simply be a convolution of the two relevant distributions. The energies are functions of only the squares of the individual particle transverse momenta, and thus play no role in the angular correlations (i.e., $\boldsymbol{k}_{j} \cdot \boldsymbol{k}_{j^{\prime}}$ ). Thus the convolution will only slightly smear out the individual impact parameters, and not influence the average pattern of their distribu- tion. If we were interested in quantitatively accurate results, we could easily include the effect of such a convolution. We return to the MPM, ignoring indirect dependence on transverse momenta.

Instead of using the $b_{j}$ 's directly in our calculation, we wish to define variables $B_{j}$ conjugate to the $Q_{j}$. By momentum conservation $Q_{j}$ is given by

$Q_{j}=\sum_{j^{\prime}=1}^{j} k_{j}$.

This allows us to relate $\boldsymbol{B}_{j}$ and $\boldsymbol{b}_{j}$. We require, in order for $Q_{j}$ and $B_{j}$ to be conjugate, that

$$
\begin{gathered}
\prod_{j=1}^{n}\left[\mathrm{~d}^{2} b_{j} \exp \left(-\mathrm{i} k_{j} \cdot b_{j}\right)\right] \delta^{2}\left(\sum b_{j}\right)= \\
K_{n} \prod_{j=1}^{n-1}\left[\mathrm{~d}^{2} B_{j} \exp \left(-\mathrm{i} Q_{j} \cdot B_{j}\right)\right] .
\end{gathered}
$$

This equation is solved (uniquely) by

$B_{j}=b_{j}-b_{j+1}$

and the proportionality constant is $K_{n}=1 / n^{2}$.

We can write the overall impact parameter (given by the last $\delta$ function in eq. (3)) in terms of the $B_{j}$ 's:

$\boldsymbol{b}=\sum_{j=1}^{n-1} \chi_{j} \boldsymbol{B}_{j}$

From the condition

$\boldsymbol{b}=\sum_{j=1}^{n}\left(p_{j} / p\right) \boldsymbol{b}_{j}$

we obtain

$\chi_{j}=\sum_{j^{\prime}=1}^{j} p_{j^{\prime}} / p$.

Eq. (6) represents a very important feature of the MPM. The natural variable is not the impact parameter $b_{j}$ but the impact parameter step $B_{j}$. The short range order assumption is that the amplitude has the form

$$
\begin{aligned}
M_{n}=Z_{n}\left(p_{j}\right) \prod_{j=1}^{n-1} F\left(Q_{j}\right) \\
\quad\left(M_{n}=Z_{n} \prod_{j=1}^{n-1} \exp \left(-R^{2} Q_{j}^{2} / 2\right)\right)
\end{aligned}
$$


where the first equality in this and the following equation is the general result, while the second is for a Gaussian distribution. This transforms to

$$
\tilde{M}_{n}=Z_{n} \prod \tilde{F}\left(B_{j}\right) ; \quad\left(\tilde{M}_{n}=Z_{n} \Pi \exp \left(-B_{j}^{2} / 2 R^{2}\right)\right)
$$

(uninteresting factors are included in $Z_{n}$ ). Thus the impact parameter steps are independent. A set of independent steps is the definition of a random walk. Thus we have derived the important result that the MPM is equivalent to a random walk in impact parameter [7].

There are two consequences we can draw from this result. We assume $b_{j-1}$ and held fixed. Then the uncertainty in $b_{j}$ depends only on the step size $\left(\Delta b_{j} \sim R\right)$ and not on the number of steps. The root mean square (rms) transverse momentum, $\sqrt{\left\langle k_{j}^{2}\right\rangle}$, is related by the uncertainty principle to the rms step size. The precise result is

$$
\begin{aligned}
& \frac{\mathrm{d} \sigma}{\mathrm{d} k^{2}} \sim \int \mathrm{d}^{2} q\left|F\left(q+\frac{1}{2} k\right) F\left(q-\frac{1}{2} k\right)\right| \\
& \mathrm{d} \sigma / \mathrm{d} k^{2} \sim \exp \left(-R^{2} k^{2} / 2\right) .
\end{aligned}
$$

From multiparticle data, the average transverse momentum is about $350 \mathrm{MeV} / c$, and the rms transverse momentum is about $430 \mathrm{MeV} / c$. We can therefore solve for the step size

$$
R^{2} \geqslant 10.9(\mathrm{GeV} / c)^{-2} \text {. }
$$

We have written this as an inequality, as in general the uncertainty principle is an inequality. If there is a rapid phase variation with $k$, the average $R^{2}$ will exceed this limit. This larger value of $R^{2}$ is the origin, in our approach, of the dependence of the elastic slope on the phases of the production amplitudes, as has been emphasized by Michejda and others [8]. In what follows we shall take it to be an equality since in our simple model it turns out that any other case is less interesting. More importantly, however, guided by the data, we will take $R$ to be independent of energy in the region of interest.

As a second consequence of the random walk, we know that the rms value of $b_{1}-b_{n}$ is proportional to the square root of the number of steps taken. The number of steps is $n-1$, where $n$ is the multiplicity.
Thus

$\left\langle\left(b_{1}-b_{n}\right)^{2}\right\rangle=(n-1) R^{2}$.

The rms impact parameter is related to $\left(b_{1}-b_{n}\right)$ by

$\left.\left\langle b^{2}\right\rangle=\left\langle\chi^{2}\right\rangle\left\langle b_{1}-b_{n}\right)^{2}\right\rangle$

where

$\left\langle\chi^{2}\right\rangle=\sum_{j=1}^{n-1} \chi_{j}^{2} /(n-1)$

and the $\chi_{j}$ are given by eq. (9). We now can calculate elastic scattering by use of eq. (3). Before we do this we make a slightly imprecise calculation. We assume for this imprecise version that multiplicities different from the average multiplicity do not matter. In this case, the elastic scattering resulting from a random walk should be Gaussian: $\operatorname{Im} \widetilde{M}_{\mathrm{el}} \sim \exp \left(-b^{2} /\left\langle b^{2}\right\rangle\right)$. This trarsforms to $\operatorname{Im} M_{\mathrm{el}} \sim \exp \left(\left\langle b^{2}\right\rangle t / 4\right)$. Putting in our expression for $\left\langle b^{2}\right\rangle$, we get

$\operatorname{Im} M_{\mathrm{el}} \sim \exp \left(\left\langle\chi^{2}\right\rangle\langle n-1\rangle R^{2} t / 4\right)$.

We have already calculated the minimum value of $R$. $\langle n\rangle$ can be taken from data. At $P_{\mathrm{LAB}}=100 \mathrm{GeV} / c$, $\langle n\rangle \approx 10$. $\left\langle\chi^{2}\right\rangle$ can also be estimated from data as explained below. The result is $\left\langle\chi^{2}\right\rangle=0.6$. From eq. (20), the slope works out to be $\left\langle\chi^{2}\right\rangle\langle n-1\rangle R^{2} / 4=15 \mathrm{GeV}^{-2}$.

We must check to see whether eq. (17) needs improvement, and calculate the value of $\left\langle\chi^{2}\right\rangle$. If neutral particles could be seen, $\left\langle\chi^{2}\right\rangle$ could be directly calculated from data. Since this is not possible, $\left\langle\chi^{2}\right\rangle$ must be estimated. The value we obtain is completely insensitive to details.

The curves we show have been calculated from the following model of the combination of data and short range order:

1) Each step is Gaussian with $R^{2}=10.9$, as we have calculated.

5) Each event is replaced by one in which the rapidities are equally spaced. The first and last particles (nucleons) have transverse mass $1 \mathrm{GeV} / c^{2}$; all other particles (pions) have transverse mass $390 \mathrm{MeV} / \mathrm{c}^{2}$ (slightly larger than the $350 \mathrm{MeV} / c$ transverse momentum).

3) Multiplicities have a Poisson distribution in $n-2$.

4) $\langle n\rangle=10$ at $P_{\mathrm{LAB}}=100 \mathrm{GeV} / c$.

From these assumptions, $\left\langle\chi^{2}\right\rangle$ is about 0.6 at 100 $\mathrm{GeV} / c$ (and rises slowly with energy). Extreme bounds 


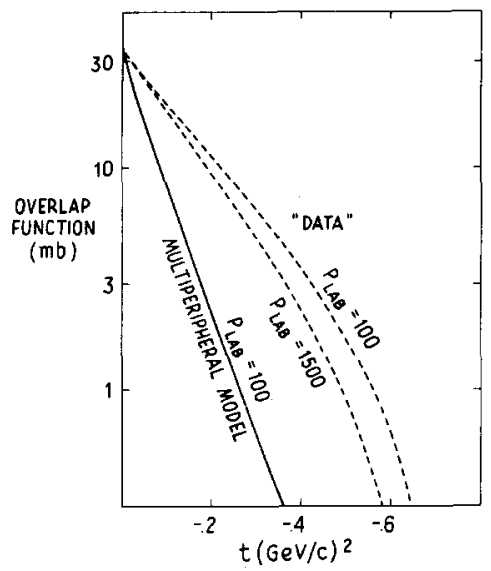

Fig. 2. A comparison of the multiperipheral model with the shortest range order possible, and the overlap function extracted from elastic scattering data. The overlap function is the sum of all inelastic contributions to the imaginary part of the elastic scattering amplitude.

on $\left\langle\chi^{2}\right\rangle$ are that leading protons have average energy fraction $\approx 0.6$, which gives $\left.\left\langle\chi^{2}\right\rangle\right\rangle 0.36$, and $\left\langle\chi^{2}\right\rangle$ is rigorously less than 1 . The calculated value is intermediate between these limits, and very insensitive to the details of the assumptions.

We compare these results with the "overlap function" [9], i.e., that part of the imaginary part of elastic scattering excluding the contribution of elastic scattering itself to unitarity. This is because elastic scattering is much larger (being Pomeron exchange) than calculated as a multiparticle process in the MPM. In order to extract the imaginary part and to calculate the overlap function from the elastic data it is necessary to make some assumption about the phase of elastic scattering. The result is not sensitive to any reasonable choice of this phase.

The comparison between the MPM and overlap function is shown in fig. 2. The MPM gives much too large a slope [10]. From eq. (17) the curve should be a straight line of slope 15 . One can easily see that eq. (17) is entirely adequate. This slope is to be compared to that of about 5 for the data. (The cross section, with slope 10 , is the square of the amplitude.) Thus this extreme short range order model must be re. jected. Our result is quite general and holds for any model satisfying eq. (10), regardless of details.

It turns out, however, that the slope is very sensi-

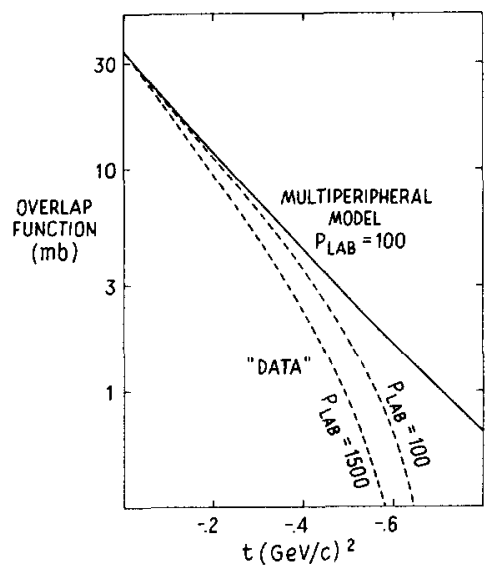

Fig. 3. A modified multiperipheral model with correlations (clustering). The correlation length is chosen to give a fit at $P_{\mathrm{LAB}}=100 \mathrm{GeV} / c$, as shown in this figure.

tive to clustering or correlations. This comes about for two reasons. First, the number of steps is smaller, so the random walk does not get as far. Second, the step size $\mathrm{R}$ might be smaller. Each particle has only a share of the transverse momentum of a cluster, plus a modest amount from the decay of the cluster. Thus the rms transverse momentum of a cluster, $1 / R$, can be significantly larger than $430 \mathrm{MeV}$. With an average cluster size somewhat less than two particles (the amount less depends on the details of calculating $R$ ), the $100 \mathrm{GeV}$ "data" can easily be fit, as shown in fig. 3 .

But, if we assume that the average cluster size, $\langle N\rangle\langle\langle n-1)$, (where $\langle N\rangle$ is the average number of steps) and the average step size $R$ are energy independent we can calculate elastic scattering at any energy. $\left\langle\chi^{2}\right\rangle$ is constant or slightly rising with energy, and the multi. plicity is rising. Therefore the model exhibits shrinkage. The results at the highest ISR energy are shown in fig. 4 . The curve is most sensitive to $\langle n\rangle$ but in any case it is much steeper than the data. The excessive shrinkage can also be exhibited by plotting an effective Regge trajectory. This is done in fig. 5, and is compared to the effective trajectory of the data. Elastic unitarity has been added in order to calculate the MPM curve. It is readily apparent that the multiperiphcral model completely fails to describe the data.

There are two ways out of this dilemma. One can assume that the ISR energies are still low energy and 


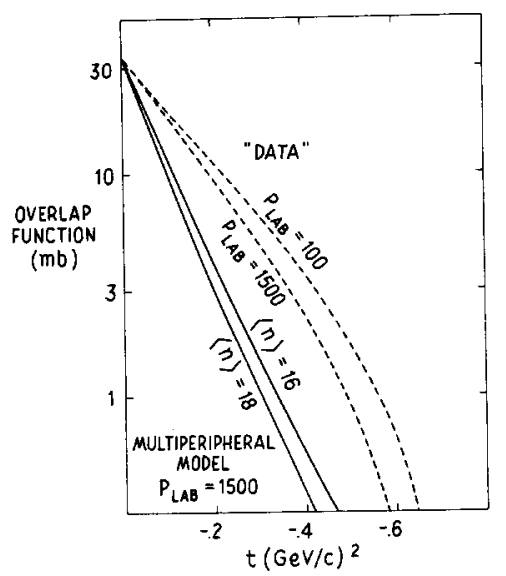

Fig. 4. The modified multiperipheral model at ISR energy. The amount of clustering is determined by the fit at $100 \mathrm{GeV}$ in fig. 3.

that the cluster size or correlation length is still growing fast enough to compensate for the increase in average multiplicity. With this assumption, one has given up any real distinction between the peripheral model and other models (except for the philosophical framework). Alternatively, one can reject the MPM, and assume long range order. Then the amplitude will depend on impact parameter as well as on the step in impact parameter. Even a modest cutoff in impact parameter can control the excessive shrinkage of the MPM.

We have so far discussed oversimplified versions of the MPM. The more detailed models which are widely studied are the ABFST model [11,2] and the CLA model $[12,3]$. The ABFST model has pairs of pions emerging from each vertex. Thus, there is a large amount of clustering, and therefore the slope will be shallow. The amount of clustering is essentially energy independent (at least at high energies where the average subenergy has reached its asymptotic value). Thus, the Regge slope will be too large. This result has been often obtained before [2].

The CLA model was explicitly constructed to include clustering. In fact, the only extremum of the amplitude (ignoring energy-momentum conservation) is a minimum at all subenergies equal. Therefore, in the CLA model, the clustering is extremely large, and the slope of elastic scattering is very flat, as has been found by direct calculation [3]. Moreover, the amount

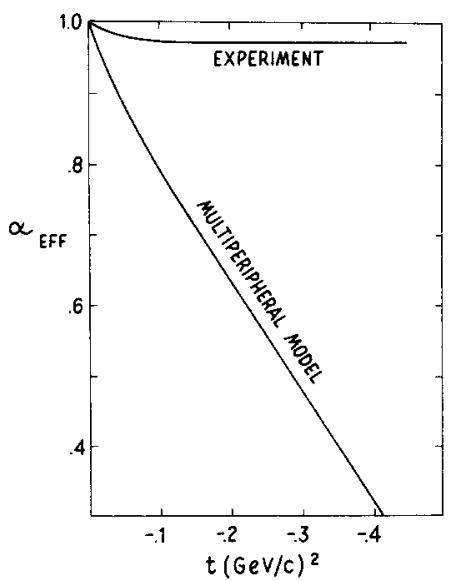

Fig. 5. The effective Regge trajectory, giving the energy dependence between $P_{\mathrm{LAB}}=100 \mathrm{GeV}$ and $1500 \mathrm{GeV}$. The slope of the multipheripheral model trajectory is four times larger than the experimental trajectory, and continues to fall, while the experimental trajectory flattens out.

of clustering increases with energy (because there are more particles to cluster) so the Regge slope is smaller than in models for which the cluster size is constant.

Summary. The main results of this paper are as follows: the multiperipheral model is given by a random walk in impact parameter. The slope of elastic scattering is given by the average step size squared (related to the average transverse momentum of produced particles by an uncertainty principle) times the average number of steps. The average number of steps times the average cluster size gives the average multiplicity. If the step size and cluster size are independent of energy, then the experimental increase in the average multiplicity gives much more shrinkage than observed in elastic scattering.

I would like to thank E.H. deGroot, G.L. Kane, E. Squires, and L. VanHove for useful discussions and correspondence.

\section{References}

[1] For a review of the multiperipheral model, see, for example, A. Pignotti, talk presented at the Symposium on High energy interactions and multiparticle production, Argonne National Laboratory, November 1970.

[2] ABFST Model: Many papers. A recent example is C.F. Chan and B.R. Webber, Phys. Rev. 5 (1972) 933. 
[3] CLA Model: L. Michejda, J. Tarnau and A. Bialas, Nuovo Cim. 56A (1968) 241.

[4] F. Henyey, Phys. Lett. 45B (1973) 363 and references therein.

[5] I would like to thank E. Squires for raising the question of the possible importance of this point.

[6] Compare, for example, the approximate formula of L. Caneschi and A. Schwimmer, Nucl. Phys. B44 (1972) 31, and Gribov (ref. [7]).

[7] This result has been previously mentioned by V.N. Gribov, JNP 9 (1969) 369. He uses only the approximate relation $b \approx b_{1}-b_{n}$.
[8] L. Michejda, Fortschritte der Physik 16 (1968) 707.

[9] L. Van Hove, Nuovo Cim. 28 (1963) 798.

[10] This result is also obtained by R.C. Hwa, Oregon preprint, January 1973.

[11] L. Bertocchi, S. Fubini and M. Tonin, Nuovo Cim. 25 (1962) 626;

D. Amati, A. Stanghellini and S. Fubini, Nuovo Cim. 26 (1962) 896.

[12] H.M. Chan, J. Loskiewicz and W.W.M. Allison, Nuovo Cim. 57A (1968) 93. 